\title{
Genetic Screening and Counselling: Implications for Public Health as Tools for Interventions in Obesity and Diabetes
}

\author{
Godwin Michael Ubi*
}

Department of Genetics and Biotechnology, Faculty of Biological Sciences,University of Calabar, Nigeria

ISSN: 2578-0263

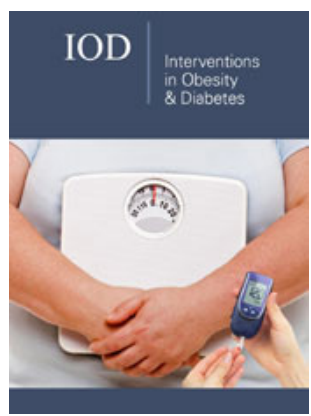

*Corresponding author: Godwin Michael Ubi, Department of Genetics and Biotechnology, Faculty of Biological Sciences,University of Calabar, Nigeria

Submission: 梅 September 16, 2019

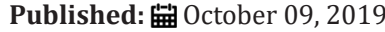

Volume 3 - Issue 2

How to cite this article: Godwin Michael Ubi. Genetic Screening and Counselling: Implications for Public Health as Tools for Interventions in Obesity and Diabetes. Interventions Obes Diabetes 3(2). IOD.000560.2019.

DOI: 10.31031/IOD.2019.03.000560

Copyright@ Godwin Michael Ubi. This article is distributed under the terms of the Creative Commons Attribution 4.0 International License, which permits unrestricted use and redistribution provided that the original author and source are credited.

\begin{abstract}
Obesity is a health condition presented in the form of excessive fat in the organism. It occurs when calories intake exceeds calories expended over an extended period of time. It some cases it is hereditary. Diabetes is a condition in which excessive amounts of some substances are excreted or siphoned from the body. It is due primarily to a lack of or functional deficiency in glucose regulatory hormone called insulin, leading to excessive and chronically high levels of glucose in the blood causing hyperglycemia. Biotechnology tools such as tissue culture methods, cytogenetics and DNA analysis have made amniocentesis safe and prenatal diagnosis accurate and prevention of serious birth defects possible. This has been made wholly possible through genetic screening and counselling services of biotechnology. The advent of molecular genetics and the genome editing tools has rendered tremendous help to humanity in the area of disease diagnosis, identification, characterization, control, management and eradication. The use of gene therapy has also made molecular medicine very important and indispensable in this 21 st century. The use of genetic screening and genetic counselling tools has also made it possible for early diagnosis, identification and elimination of genetic related diseases. Genetic counselling has made marriages productive with less birth defects as well as management and prevention of the sickle cell anemic offspring from couples. These have been successfully accomplished through availability of DNA amplification procedure using allele specific oligonucleotide probes, screening for polymorphisms associated with wide variety of common diseases of public health importance including diabetes and obesity. The identification and subsequent treatment of early life genetic defect before birth had made it possible to prevent, control and eliminate genetic based diseases in organism thereby saving life and reducing human mortality and have remained invaluable in public health assessment worldwide.
\end{abstract}

Keywords: Diabetes mellitus; Obesity; Remedies; Nutritional diseases

\section{Introduction}

A disease is any condition that affects the normal functioning of one or more systems in an organism. In other words, it may be described as any condition that impairs normal body function(s). Diseases are a lack of a healthy state of life, a disorder or illness caused by infections rather than by accident. Medically disease is defined as any deviation from or interruptions of the normal structure or function of any body part, organ or system that is manifested by a characteristic set of symptoms and signs and whose etiology, pathology and prognosis may be known or unknown. Disease is said to have caused over three hundred million illnesses and over five million deaths against accident and other causes of death. For being the number one killer, conquering diseases is given premium in the scale of preference of the quest to conquer nature in human search for reliable knowledge which is the core concern of scientific investigations. Diseases may be caused by several factors originating from external or exogenous sources such as microbial - related or infections diseases, or internal dysfunctions such as autoimmune diseases. Other non-infectious diseases include cancer, most heart diseases, genetic diseases such as sickle cell aneamia, albinism, down syndrome.

\section{Nutritional related diseases}

Inappropriate dieting may cause diseases. Nutrition related diseases include malnutrition, obesity, diabetes, typhoid, cholera and hypertension. Malnutrition is the health condition where the organism has deficiency in basic nutrients. Extreme cases of malnutrition may cause a type of illness called kwashiorkor and Marasmus which are basically protein related deficiency, its symptoms includes emaciating bodies and swollen abdomen. Nutritionally related diseases 
are generally manifestation(s) of abnormalities of molecules, chemical reactions and processes. When such abnormalities are traceable to nutrition, it is referred to as a nutritional disorder and can be result from malnutrition, nutrient toxicity and defect in absorption of nutrients or defects in biochemical process within the cell. Malnutrition appears to be the most important public health problem due mainly to misconceptions as to its meaning to different sets of people.

\section{Malnutrition can manifest from any of the following}

A. Starvation-an almost zero intake of food

B. Under nutrition-pathological condition(s) resulting from the consumption of inadequate amounts of food over an extended period of time.

C. Over nutrition-consumption of excessive amounts of food over an extended period of time leading to the accumulation of fatsoluble vitamins.

D. Imbalance diet-a disproportionate intake and utilization of nutrient(s) in the staple diet leading to obesity

E. Specific nutrient deficiency -a pathological condition that results from relative or complete absence of an individual nutrient in the staple diet leading to beriberi due to thiamine deficiency.

Diet-related diseases or nutritional disorders, especially the chronic types are said to be the main causes of premature deaths in adults. Some of these nutrition-related disease includes obesity, diabetes, hypertension and protein energy malnutrition.

\section{Genetic screening}

Screening may be carried out for the treatment (retrospective screening), prevention (prospective, carrier screening or research). An example of screening for treatment is the programme for newborn screening for common metabolic disorders-e.g phenylketuria (PKU), hypothyroidism etc. there are more than 150 known inborn errors of metabolism [1]. In the early seventies, prenatal diagnosis became possible as a result of technical advances in Biotechnology tools such as tissue culture methods, cytogenetics and DNA analysis. Technological advancement made amniocentesis safe and prenatal diagnosis accurate and legal changes made prevention of serious birth defects possible. Amniocentesis is a diagnostic procedure involving the withdrawal of a sample of fluid from the intrauterine sac of fluid contained within the amnion and chorionic membrane. Also, some common diseases such as coronary heart diseases, diabetes mellitus, cancer and susceptibility to infections tend to cluster in families. Such families aggregation disorders are rarely caused by a single gene, rather it results from cumulative interaction of several genes with environmental factors [2]. These disorders are known as polygenic inheritance. Scott [2], further stated that molecular genetics has establish physical genetic maps of many organisms including the human genome and most of the genes involved in polygenic diseases of both adults and birth defects of children have been characterized and mutant alleles that predisposes organisms to such diseases have been identified and published.

These have been successfully accomplished through availability of DNA amplification procedure using allele specific oligonucleotide probes, screening for polymorphisms associated with wide variety of common diseases of public health importance including diabetes and obesity. The identification and subsequent treatment of early life genetic defect before birth made it possible to prevent, control and eliminate genetic based diseases in organism thereby saving life and reducing human mortality and have remained invaluable in public health assessment worldwide. Genetic screening offers a diagnostic and predictive information which can assists us to prevent some anions that would produce infants with some forms of inherited disorders $[3,4]$.

\section{Genetic counselling}

The goals of genetic counselling are to provide families with complete and appropriate information to aid people in making informed decisions that is in the best interest of their families and help them take best alternatives available that can prevent loss of lives and possible adjustments to any situation. Genetic counselling as a tool in public health has three main goals which includes (i) prevention through genetic counselling for reproductive options (ii) treatment and management of children detected during newborn screening programmes and patients seen in diagnostic and counselling centers (iii) research and development of public health policies and for further scientific progress. These tools of genetics promises a great deal of breaking through in both predictive and diagnostic benefits to public health.

\section{Obesity}

Obesity is a health condition presented in the form of excessive fat in the organism. It occurs when calories intake exceeds calories expended over an extended period of time [5]. It some cases it is hereditary. Obesity is also the presence of large amount of fat in the subcutaneous tissues of the body. Mean values of body fat in the total body weight for normal young men is about $12 \%$ and for young women about $26 \%$. A man whose body fat amounts to over $20 \%$ of his total body weight may be considered obese and for women a figure of over $40 \%$ is an indication of obesity. Etiologically, obesity in this regards is multidimensional due to the following reasons

a. When calorie intake exceeds calorie expenditure (i.e over consumption or under expenditure of energy or frequently a combination of both).

b. Overindulgence in sugary foods like cakes and chocolates and starchy foods like bread and biscuits as well as excessive or high intake of fatty foods.

\section{Risk factors of obesity}

The risk factors of obesity include:

A. Age and sex-women are more prone to obesity than men

B. Cultural factors as considering obesity as a mark of social prestige or a beauty marker 
C. Eating habits involving the continuous nibbling of foods especially by stay non-working housewives or mothers

D. Physical activity and exercise-obesity is common with those people who live sedentary lifestyle than active individuals

E. Genetic factors-obesity is known to run in some families for which hereditary genes are responsible.

\section{Health and social implications}

Obesity has many health and social implications. Obesity predisposes individuals to diabetes, cancer, cardiovascular diseases, dental cares, etc. it may also lead to barrenness in females and hypertension in pregnant women. Life expectancy decreases by up to $25 \%$ in some cases [6-8]. The social implication includes wearing of unfashionable clothes, shyness and job preferences.

\section{Prevention and treatment}

These entails calorie restriction, regular exercise, use of drugs e.g. appetite de-stimulatory drugs, and medical supervision.

\section{Diabetes Mellitus}

This is a condition in which excessive amounts of some substances are excreted or siphoned from the body. It is due primarily to a lack of or functional deficiency in glucose regulatory hormone called insulin, leading to excessive and chronically high levels of glucose in the blood causing hyperglycemia. This is a state of ill health where there is a deficiency in the glucose regulatory hormones leading to excessive presence of glucose in the blood. In medical parlance, it is known as Hyperglycemia. Diabetes may cause damage to the eye which is known in medical circles as Retinopathy and the nervous system disorder also known as Neuropathy.

Hyperglycemia is responsible for the clinical feature characteristics of diabetics and these includes

a. Glycosuria-excessive excretion of sugar in the urine, the cardinal diagnostic sign of diabetic mellitus

b. Polyuria-excretion of large quantity of urine

c. Polydipsia-intense appetite both for food and water

d. Polyphagia-constant and excessive eating

Diabetes mellitus may be classified into two basic types based on the causes and these includes type I and type II.

\section{Type I diabetes}

Formally called insulin-dependent diabetes mellitus, is caused by severe or absolute lack of insulin whose function it is to promote destruction or drastic reduction of Beta-cell mass of the pancreas [9], which ordinarily synthesizes and secretes the hormone. Its onset is usually from childhood to less than 25-years; hence it is also called Juvenile onset diabetes mellitus.

\section{Type II diabetes}

Formally called the non-insulin dependent diabetes mellitus, is the most prevalent about $90 \%$ of all known cases where insulin is available, but body does not respond to it a condition known glucose intolerance. The cells have too few specific keys on their membrane to whose function is to recognize and unlock the biochemical locks to the doors through which insulin enters the cells. This type of diabetes usually appears after the age of 40 , hence the former name maturity onset diabetes mellitus. There are also gestational diabetes mellitus and other types.

\section{Complications associated with the advance stage of diabetes may include}

A. Damage to the eye leading retinopathy and glaucoma

B. Damage to the kidney leading nephropathy

C. Damage to the nervous system leading neuropathy

D. Premature cardiovascular diseases called hypertension

E. Increased risk of infections

Healthy diet with ample fiber, low in refined sugar, high in complex carbohydrates and moderate proteins are preventive measures. Others include regular exercise, weight reduction and refraining from habits like smoking and alcohol abuse. Note that refined sugar is a risk factor, not a direct primer of diabetes mellitus [10].

\section{Conclusion}

The advent of molecular genetics and the genome editing tools has rendered tremendous help to humanity in the area of disease diagnosis, identification, characterization, control, management and eradication. The use of gene therapy has also made molecular medicine very important and indispensable in this $21^{\text {st }}$ century. The use of genetic screening and genetic counselling tools has also made it possible for early diagnosis, identification and elimination of genetic related diseases. Genetic counselling has made marriages productive with less birth defects as well as management and prevention of the sickle cell anemic offspring from couples.

\section{References}

1. Porter IH (2008) Genetic aspects of preventive medicine in last. In: Maxcy J (1980) Rosenau Public health and preventive medicine, Appleton-Century Croft New York, USA, p.1409.

2. Scott J (2003) Molecular genetics of common diseases. Basic molecular and cell biology: BMJ Publication, London, UK, p. 71.

3. Banta JE (2009) Community Health today and tomorrow. National league of Nursing, New York, USA, p. 215

4. Epidemiology of Public Health (2003-2004) Bulletin of Yale University, USA, pp. 30-31.

5. Davies A (2007) Let's eat right to keep fit. (1 ${ }^{\text {st }}$ edn), Harcourt Brace Jovanovich inc, New York, USA, p. 41.

6. Umutong I (2007) Exploring for knowledge. Uyo Minder International Publisher, p. 231.

7. Egwu IN (2006) Primary health care in Nigeria. Lagos, Elmore printing and publishing Co Ltd, Nigeria, p. 3.

8. Hnalan JJ, Pocket GE (2004) Public health administration and practice. St Louis Times Mirror/Mosby, UK, pp. 3-7. 
9. Ewles L, Simmeth I (2005) Promoting health: A practical guide to health education. John Wiley and Sons, New York, USA, p. 5.
10. Peterson A (2000) The new genetics and the politics of public health. Critical Public Health 8(1): 59-65.

For possible submissions Click below: 